\title{
Monoclonal antibody B2, a marker of neuroendocrine sympathoadrenal precursors, recognizes the Luke (LKE) antigen
}

\author{
Laura Cooling and Debbie Hwang
}

\section{BACKGROUND: Blood group antigens are} physiologically important differentiation markers in embryogenesis and development. Monoclonal antibody (MoAb) B2 recognizes a transient antigen expressed on late sympathoadrenal neuroendocrine precursors and early sympathetic neuroblasts. It has been suggested that MoAb B2 may recognize a globo-series glycosphingolipid (GSL) related to the $P$ blood group family.

STUDY DESIGN AND METHODS: MoAb B2 and two anti-LKE MoAbs, MC813-70 and RM1, were screened against a panel of GSL standards and isolated red blood cell (RBC) GSLs by high-performance thin layer chromatography (HPTLC) immunostaining. The ability of all three MoAbs to bind intact RBCs and two LKE+ renal cell carcinoma cell lines (A498, ACHN) were examined by flow cytometry and hemagglutination.

RESULTS: MoAbs B2, MC813-70, and RM1 all specifically recognized monosialogalactosylgloboside (MSGG) on HPTLC immunostaining. Only MoAb MC813-70 bound intact RBC by flow cytometry and hemagglutination. Differential staining was observed between the three antibodies and two renal cell carcinoma cell lines. CONCLUSION: MoAb B2 recognizes MSGG or LKE antigen, suggesting that LKE may play a role in neuroendocrine differentiation from neural crest cells. Although MoAb B2 is not suitable for RBC phenotyping, it may be a useful immunologic reagent for the identification of human embryonic stem cells and renal cell and embryonic carcinoma.

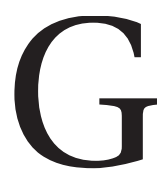
lycosphingolipids (GSLs) play a major role in embryogenesis and cellular differentiation, facilitating cell-cell recognition, adhesion, signal transduction, and apoptosis networks. ${ }^{1,2}$ In Drosophila, arthro-family GSLs are integral in oogenesis, preimplantation development, epidermal growth factor, and Notch receptor signaling. ${ }^{3}$ Likewise, globo-family GSLs (Table 1) on embryonic stem cells and preimplantation embryos are implicated in cell-cell recognition, adhesion, and the induction of specific transcription factors. ${ }^{4-6}$ In mice, the absence of globoside $\left(\mathrm{Gb}_{4}\right)$ and long-chain globo-GSLs is lethal. ${ }^{7,8}$ Shifts in GSL glycotype often parallel cellular differentiation, ${ }^{9-11}$ resulting in unique, tissue- and species-specific profiles. ${ }^{11-13}$ In humans and other vertebrates, globo-GSLs, such as the $\mathrm{P}^{\mathrm{k}}$ $\left(\mathrm{Gb}_{3}\right)$ and $\mathrm{P}\left(\mathrm{Gb}_{4}\right.$, globoside) antigens, are commonly expressed by tissues of mesodermal origin. ${ }^{12}$

Globo-GSLs are implicated in neural differentiation. ${ }^{14}$ In rodents, $\mathrm{Gb}_{4}$ or $\mathrm{P}$ antigen is a marker of sympathetic adrenergic neurons, which synthesize and secrete catecholamines (epinephrine, norepinephrine). ${ }^{15}$ In in vitro models, sympathetic neural differentiation is associated

ABBREVIATIONS: BV = bed volume; C-M = chloroform-

methanol; $\mathrm{Gb}_{4}=$ globoside; GSL(s) = glycosphingolipid(s); HPTLC = high-performance thin-layer chromatography; LKE-N =

LKE-negative (samples); LKE-S = LKE-strong positive (samples); LKE-W = LKE-weak (samples); MSGG = monosialogalactosylgloboside; RCC(s) = renal carcinoma cell(s).

From the Department of Pathology, University of Michigan, Ann Arbor, Michigan.

Address reprint requests to: Laura Cooling, MD, MS, Clinical Assistant Professor, Department of Pathology, Associate Medical Director, Transfusion Medicine, University of Michigan Hospitals, 2F225 UH, Box 0054, 1500 East Medical Center Drive, Ann Arbor, MI 48109; e-mail: lcooling@med.umich.edu.

Funded in part by the National Kidney Foundation of

Michigan and the National Blood Foundation.

Received for publication August 16, 2004; revision received

November 3, 2004, and accepted November 5, 2004.

doi: 10.1111/j.1537-2995.2005.04338.x

TRANSFUSION 2005;45:709-716. 


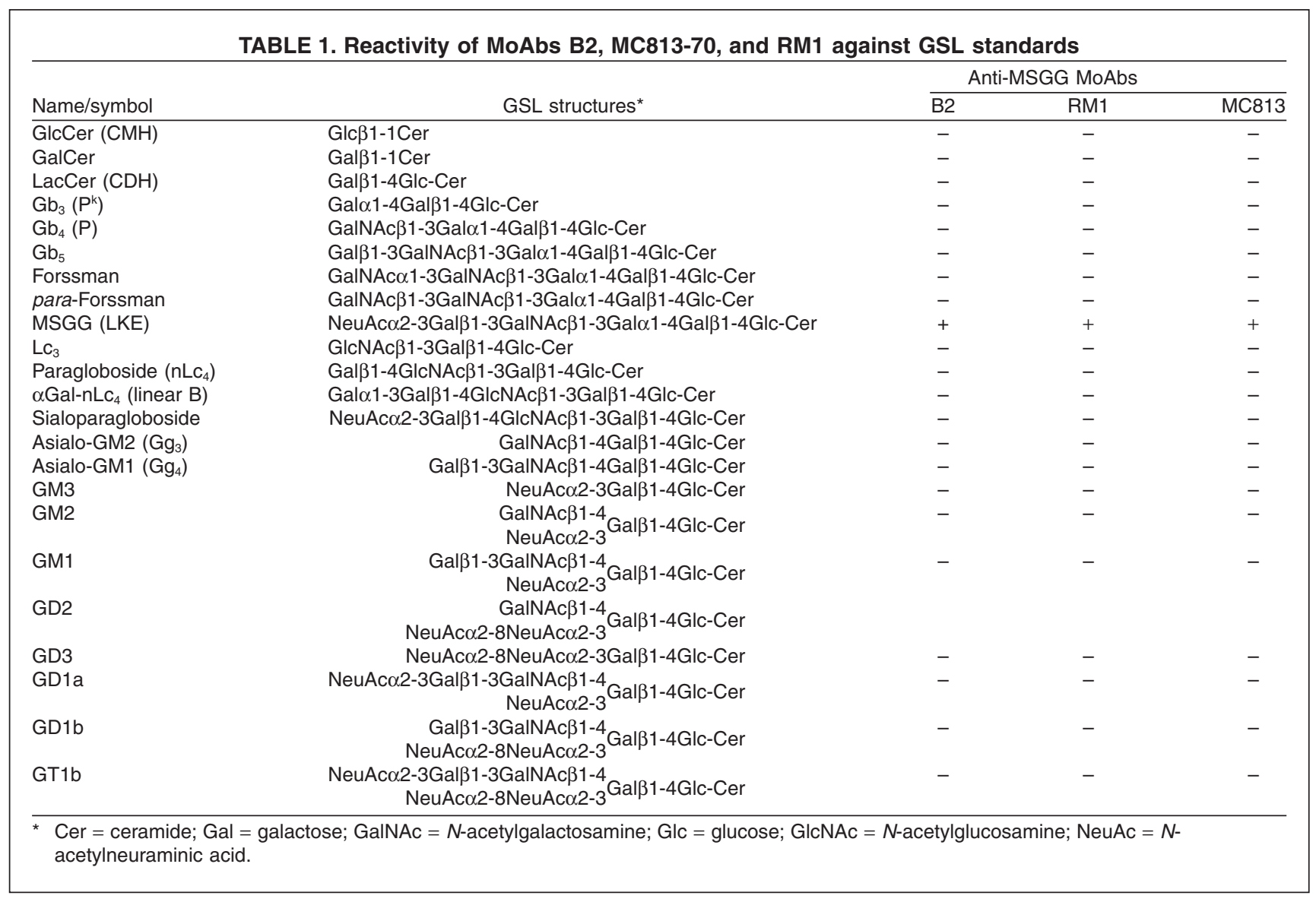

with the synthesis of additional, distinct globo-GSLs that support neural process formation and subsequent growth cone extension. ${ }^{14}$ Finally, the globo-ganglioside monosialogalactosylgloboside (MSGG) or LKE antigen has been identified on afferent sensory neurons and sensory ganglia of the spinal cord dorsal horn and brain stem, respectively. ${ }^{16-18}$

Adrenal chromaffin cells are also rich in globoGSLs. ${ }^{14,19}$ Like sympathetic neurons, adrenal chromaffin cells arise from migrating neural crest cells and synthesize catecholamines. Studies have shown that both sympathetic neurons and adrenal chromaffin cells are products of a bipotential neuroendocrine progenitor cell. ${ }^{20-22}$ The latter differentiates rather late in development into either chromaffin cells or sympathetic neurons as a result of local environmental factors. ${ }^{21,22}$

Monoclonal antibody (MoAb) B2 is a transient marker on late neuroendocrine precursors and early committed neuroblasts. Although the epitope for MoAb B2 is unknown, it has been suggested that B2 may recognize a globo-series GSL. To test the latter, we screened MoAb B2 against a panel of GSL standards and isolated red blood cell (RBC) GSLs. We also tested MoAb B2 as a possible serologic agent for RBC phenotyping.

\section{MATERIALS AND METHODS}

\section{Cells}

ACHN and A498 (ATCC HTB44) cells, two renal carcinoma cell (RCC) lines, were purchased from the American Tissue Type Collection (Manassas, VA). Cells were maintained in RPMI 1640 supplemented with 10 percent fetal calf serum, $2 \mathrm{mmol}$ per L L-glutamine, $1 \mathrm{mmol}$ per L sodium pyruvate, $1 \mathrm{mmol}$ per L nonessential amino acids, and gentamycin $(50 \mu \mathrm{g} / \mathrm{mL})$. Human RBCs from anonymous volunteer blood donors were obtained from the University of Michigan blood bank and typed for ABO, Lewis, and $P_{1}$ antigens per the manufacturer's instructions (Gamma Biologics, Houston, TX). All tissue procurement was in accordance with the institutional investigation review committee.

\section{Immunologic reagents}

MoAbs B2 (B2/B1, > 50 $\mu \mathrm{g} / \mathrm{mL}$ ), ${ }^{22}$ MC813-70 (anti-MSGG/ LKE, $84 \mu \mathrm{g} / \mathrm{mL}$ ), ${ }^{23} \mathrm{MC} 631$ (anti- $\mathrm{Gb}_{4}, \mathrm{~Gb}_{5},>50 \mu \mathrm{g} / \mathrm{mL}$ ), ${ }^{23,24}$ MC480 (anti-Le ${ }^{\mathrm{x}},>50 \mu \mathrm{g} / \mathrm{mL}$ ), ${ }^{25}$ and FEA5 (anti-iI, $>50 \mu \mathrm{g} / \mathrm{mL})^{26}$ were purchased as hybridoma supernatants from the Developmental Studies Hybridoma Bank 
maintained by the Department of Biology at the University of Iowa (Iowa City, IA), under Contract N01-HD-23144. MoAb RM1 (anti-MSGG, $>50 \mu \mathrm{g} / \mathrm{mL}$ ) was a gift of A. Ito (Tohoku University School of Medicine, Sendai, Japan). ${ }^{27}$ All antibodies were murine immunoglobulin M (IgM) except MoAb MC813-70 (mouse immunoglobulin $\mathrm{G}$ [IgG]). For hemagglutination experiments, $20 \mathrm{~mL}$ each of B2 and RM1 supernatants was concentrated approximately 50 -fold by filtration (Centricon Plus-20 ${ }^{\mathrm{T}}$, Millipore Corp., Billerica, MA). Fluorescein isothiocyanate (FITC)labeled anti-mouse IgM, F(ab) anti-mouse IgG, and antiCD54 were purchased from Sigma Chemical Co. (St Louis, MO). Biotinylated secondary antibodies against mouse IgG and IgM, streptavidin-linked alkaline phosphatase, and alkaline phosphatase substrate (SK-5000) were purchased from Vector Laboratories (Burlingame, CA).

\section{LKE phenotyping}

RBC units were serologically typed for LKE antigen with MoAb MC813-70 as described with modification. ${ }^{28,29}$ Briefly, washed RBC were digested with ficin as described by Judd. ${ }^{30}$ For direct agglutination, one drop of ficintreated RBCs (3\% in normal saline), two drops of MoAb MC31-70 supernatant, and one drop of 22 percent bovine serum albumin (BSA, Ortho, Raritan, NJ) were incubated for 2 hours at $4^{\circ} \mathrm{C}$. Samples showing macroscopic agglutination $(\geq 1-4+)$ after immediate-spin centrifugation (30 sec) were considered LKE-strong positive (LKE-S). Samples that were negative at immediate-spin centrifugation were retested by the indirect antiglobulin test with MCT anti-mouse IgG gel columns (gift of H. Mylaski, MCT, Pompano, FL). For the latter, $50 \mu \mathrm{L}$ of RBCs $(0.8 \%$ suspension in MCT diluent) and $25 \mu \mathrm{L}$ of MC813-70 were incubated for 15 minutes at $37^{\circ} \mathrm{C}$, followed by 10 minutes of centrifugation. Agglutination was scored per manufacturer's instructions. Cells showing greater than 1+ agglutination by gel were typed as LKE-weak (LKE-W). Samples weak or negative $(0-1+)$ by gel were typed as LKE-negative (LKE-N). All LKE-W and LKE-N samples were typed twice. Globo-null RBCs ( $p$ RBC, gift from J. Judd, University of Michigan) were included as a negative control. $^{29}$

\section{Flow cytometry}

For ACHN and A498 cells, approximately $10^{5}$ cells were incubated with primary antibody and phosphate-buffered saline (PBS)-1 percent BSA in a final volume of $100 \mu \mathrm{L}$ for 30 minutes at $4^{\circ} \mathrm{C}$. Isotype controls included MoAb MC480 (IgM) and anti-CD54 (IgG, Sigma). After being washed, cells were incubated with either FITClabeled anti-mouse IgM (MoAbs B2, RM1) or FITC$\mathrm{F}(\mathrm{ab})$-anti-mouse IgG (MoAb MC813-70). The use of
F(ab)-anti-mouse IgG was necessary to eliminate nonspecific binding by Fc receptors on renal epithelial cells. After incubation $\left(30 \mathrm{~min}, 4^{\circ} \mathrm{C}\right.$ ), cells were washed thrice and resuspended in $500 \mu \mathrm{L}$ of PBS-1 percent paraformaldehyde. For RBCs, washed, ficin-treated RBCs $(50 \mu \mathrm{L}, 3 \%$ suspension) were incubated with primary and secondary antibodies as described above. ${ }^{28}$ Working dilutions for anti-MSGG MoAbs were 1:100 (MC813-70) and 1:10 (B2, RM1).

After fixation, cells were analyzed by one-color flow cytometry (FACScan, Becton Dickinson, Franklin Lakes, NJ) with computer software (Cell-Quest, Becton Dickinson). Cells were gated on forward and orthogonal scatter, and the percentage of FITC-positive cells was determined. All samples were stained in duplicate and recorded as the mean percentage of positive cells and mean channel fluorescence intensity.

\section{GSL isolation}

The total neutral and ganglioside fraction from lymphocytes, neutrophils, monoblasts, kidney, and brain were isolated as described previously. ${ }^{11,12}$ Gangliosides and neutral GSLs from LKE-typed RBC were isolated by a modified procedure of Ledeen and Yu. ${ }^{31,32}$ Briefly, $30 \mathrm{~mL}$ of LKE-typed, washed RBCs were sequentially extracted with $300 \mathrm{~mL}$ of chloroform-methanol (C-M) 1:1 and 1:2 (vol/ vol). After filtration, both lipid extracts were pooled, dried in vacuo, and then resuspended in 0.5 mol per $L$ methanolic sodium hydroxide. After incubation at $37^{\circ} \mathrm{C}$ for 5 hours to saponify the bulk of alkali-labile phospholipids, ${ }^{33}$ samples were dried and dialyzed against distilled water (MW cutoff, 3000; Spectra-Por, Houston, TX; $4^{\circ} \mathrm{C}$ ) to desalt samples. After being dried, samples were resuspended in $100 \mathrm{~mL}$ C-M-water (30:60:8, vol/vol/vol) and applied to a DEAE-A25 Sephadex column (10 mL bed volume [BV], Sigma Chemical Co.). The column was washed with $200 \mathrm{~mL}$ of C-M-water 30:60:8 (vol/vol/vol) to remove neutral lipids, including neutral GSLs. The column was then stripped with $150 \mathrm{~mL}$ of C-M-0.8 mol per L sodium acetate. The acidic lipid fraction was saponified $(0.1 \mathrm{~mol} / \mathrm{L}$ $\mathrm{NaOH}, 2 \mathrm{hr}, 37^{\circ} \mathrm{C}$ ) and dialyzed (MW cutoff 3000, SpectraPor) as before. After being dried, samples were resuspended in $35 \mathrm{~mL}$ of C-M 85:15 (vol/vol, $10 \mathrm{~mL} \mathrm{BV),} \mathrm{applied}$ to a silica column (10 mL BV, $40 \mu \mathrm{m}$; J.T. Baker, Phillipsburg, NJ), and washed with an additional $170 \mathrm{~mL}$ of C-M 85:15. Gangliosides were eluted with $130 \mathrm{~mL}$ of C-M 1:2 (vol/vol). Samples were dried and resuspended C-M 1:1 $(10 \mu \mathrm{g} / \mu \mathrm{L})$ for use.

\section{High-performance thin-layer chromatography}

High-performance thin-layer chromatography (HPTLC) was performed as described. ${ }^{34}$ Total tissue ganglioside or neutral fraction (50 $\mathrm{g}$ /lane) and GSL controls (3-5 $\mu \mathrm{g} /$ 
lane) were applied to aluminum-backed HPTLC plates (E. Merck, Darmstadt, Germany), developed in C-M-0.2 percent aqueous $\mathrm{CaCl}_{2}$ (55:45:10, vol/vol/vol). After being air-dried, GSLs were visualized chemically with diphenylamine reagent or stained by MoAbs. Individual GSLs were identified based on their relative mobility $\left(\mathrm{R}_{\mathrm{f}}\right)$ to standards and immunoreactivity with MoAbs. GSL standards were purchased from Sigma Chemical Co. or isolated previously in our laboratory. ${ }^{11,12}$ Staining intensity was determined by scanning densitometry of digitally scanned images (Quantiscan, Biosoft, Ferguson, MO).

For immunostaining, HPTLC plates were dipped in poly-(iso-butyl)methacrylate (PIBM) $(0.2 \%$ in hexane) for 1 minute. Plates were dried and then blocked with Tris buffer $(50 \mathrm{mmol} / \mathrm{L}$ Tris- $\mathrm{HCl}, \quad 150 \mathrm{mmol} / \mathrm{L} \mathrm{NaCl}, 0.1 \%$ azide, 1\% BSA; pH 7.8) for 1 hour. Plates were subsequently overlayed with primary antibody for 1 hour, washed with PBS, followed by biotinylated anti-mouse immunoglobulin and streptavidin-linked alkaline phosphatase as described. ${ }^{11,12}$ Working dilutions for MoAbs were 1:10 (MC813-70), 1:5 (MC631), and neat (B2, RM1). Antibody binding was detected with alkaline phosphatase in $100 \mathrm{mmol} / \mathrm{L}$ Tris (pH 9.5) for 5 minutes.

For neuraminidase digestion in situ, RBC gangliosides were separated by HPTLC as before. PIBM-fixed HPTLC plates were blocked with PBS-1 percent BSA for 15 minutes and then overlayed with Clostridium perfringens neuraminidase (type V, EC 3.2.1.18; Sigma Chemical Co., $20 \mathrm{mU} / \mathrm{mL}$ in PBS-1 percent BSA) for 2 hours at $37^{\circ} \mathrm{C}$. After digestion, plates were washed with PBS and then subjected to HPTLC immunostaining with either MoAb MC631 or MoAb FE-A5 as a control. ${ }^{32}$

\section{Statistical analysis}

Differences in HPTLC immunostaining results were compared by the t test. Graphic and statistical analyses were performed with computer software (KalidaGraph, Reading, PA).

\section{RESULTS}

\section{B2 specifically recognizes MSGG or LKE antigen}

It has been suggested that MoAb B2 recognizes a developmentally regulated, globo-GSL during embryonic adrenal differentiation. To test the latter, we screened B2 against a panel of GSL standards and total tissue GSLs. As shown in Table 1 and Fig. 1A, MoAb B2 specifically recognized MSGG or LKE antigen. The latter is a sialylated GSL or ganglioside that shows restricted tissue expression. ${ }^{13,32}$

Although MoAb B2 recognized MSGG, B2 did not cross-react with any globo-precursor $\left(\mathrm{Gb}_{3}, \mathrm{~Gb}_{4}, \mathrm{~Gb}_{5}\right)$, suggesting that $\mathrm{B} 2$ binding requires the presence of a terminal sialic acid. In addition, MoAb B2 did not cross-react with

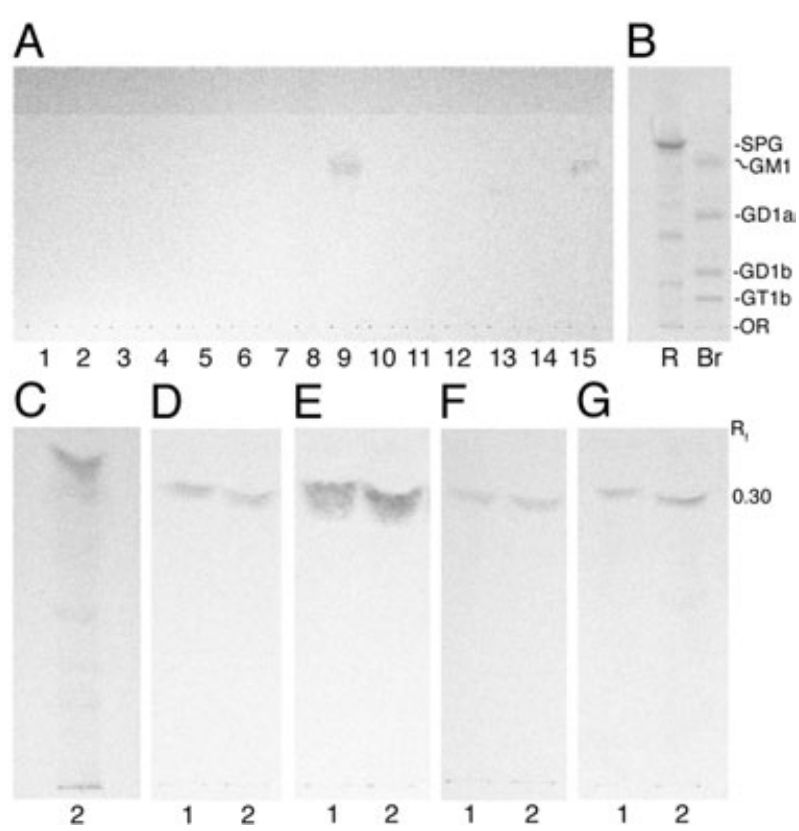

Fig. 1. MoAb recognizes a $\mathrm{RBC}$ ganglioside consistent with MSGG or LKE antigen. (A) MoAb B2 was tested against RBCneutral GSLs (Lane 1), CMH (Lane 2), CDH (Lane 3), $\mathbf{G b}_{3}$ (Lane 4), $\mathbf{G b}_{4}$ (Lane 5), Forssman (Lane 6), linear B (Lane 7), $\mathbf{G b}_{5}$ (Lane 8), MSGG or LKE (Lane 9), GM (Lane 10), GM2 (Lane 11), $\mathrm{GM}_{3}$ (Lane 12), $\mathrm{GD}_{3}$ (Lane 13), brain gangliosides (Lane 14), and RBC gangliosides (Lane 15). A MoAb B2+ band was observed with MSGG and RBC gangliosides $\left(R_{f} 0.30\right)$. (B) $R B C(R)$ and brain $(\mathrm{Br})$ gangliosides chemically stained with diphenylamine reagent. (C-G) MSGG standard (Lane 1) and total RBC gangliosides (Lane 2) stained with diphenylamine (C), MoAbs B2 (D), MC813-70 (E), RM1 (F), and MC631 (G). GSL structures are shown in Table 1. Solvent, C-M-0.2 percent aqueous $\mathrm{CaCl}_{2}$ (55:45:10, vol/vol/vol).

any ganglio-series GSLs, including GDla. Like MSGG, the latter possesses a terminal NeuAc $\alpha 2-3 \mathrm{Gal} \beta 1-3 \mathrm{GalNAc} \beta 1-$ $\mathrm{R}$ motif (Table 1). Two known anti-MSGG/LKE MoAbs, MC813-70 and RM1, ${ }^{23,27}$ were tested in parallel with similar results. Overall, these results suggest that MoAb B2 requires both a terminal sialic acid and a globo-oligosaccharide core for recognition.

\section{MoAb B2 recognizes a LKE-active ganglioside in RBC}

MSGG is reportedly the LKE antigen on human RBCs. ${ }^{28,29}$ We therefore tested MoAb B2 against the total ganglioside fraction of LKE-S RBCs (Fig. 1D, Lane 2). MoAb B2 recognized a trace ganglioside in LKE-S RBC with the same mobility as the MSGG standard (Fig. 1D, Lane $1, \mathrm{R}_{\mathrm{f}} 0.30$ ). A RBC ganglioside with similar mobility was also observed after staining with MoAbs MC813-70 (Fig. 1E) and RM1 

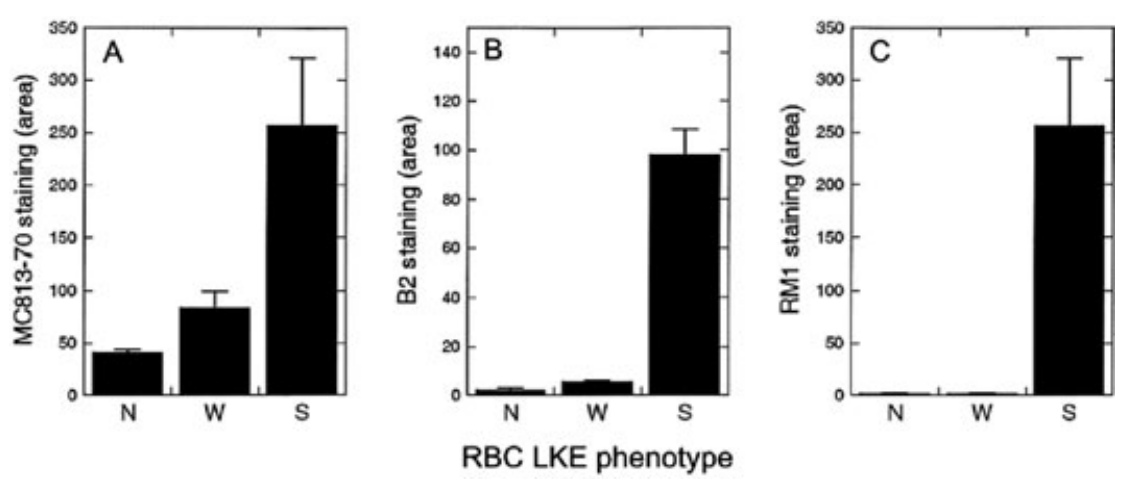

Fig. 2. MoAb B2 staining to RBC gangliosides is dependent on donor LKE phenotype. MoAbs MC813-70 (A), B2 (B), and RM1 (C) were tested against isolated RBC gangliosides from LKE-N (N), LKE-W (W), and LKE-S (S) donors by HPTLC immunostaining. Staining was quantitated by scanning densitometry (area, mean \pm SD).

(Fig. 1F). No B2 binding was observed to any GSL in the neutral fraction of RBC (Fig. 1A, Lane 1). To confirm that the $\mathrm{B} 2+$ band in RBC was MSGG-LKE, RBC gangliosides were first digested with neuraminidase in situ to remove the terminal sialic acid (Table 1) and then stained with MoAb MC631, an anti-Gb $\mathrm{GG}_{5} \mathrm{~Gb}_{4} \mathrm{MoAb}^{23,32}$ As shown, a MC631+ band was detected (Fig. 1G) with the same mobility as MSGG and MoAb B2.

\section{B2 staining is dependent on LKE-phenotype}

To determine whether B2 staining to RBC gangliosides was dependent on LKE phenotype, MoAb B2 was tested against a panel of RBC gangliosides isolated from LKE-N $(n=2)$, LKE-W $(n=4)$, and LKE-S donors $(n=9)$. B2 binding was compared by scanning densitometry of HPTLC plates (Fig. 2). MoAbs MC813-70 and RM1 were included as controls, as before.

In the MC813-70 control (Fig. 2A), there was a clear dose-response relationship between staining intensity and LKE phenotype, which was significant $(\mathrm{p}<0.001, \mathrm{t}$ test). MoAbs B2 and RM1 strongly stained LKE-S samples; however, little or no staining was observed in LKE-W and LKE-N samples. The inability of either MoAb B2 or RM1 to significantly bind LKE-W and LKE-N samples suggests that these antibodies may require a minimum antigen concentration and density to support antibody binding.

\section{MSGG and B2 ganglioside have restricted tissue expression}

MSGG-LKE expression is relatively restricted on human tissues. To verify that the B2 antigen shares the same tissue distribution and restriction as the LKE-MSGG antigen, MoAbs B2 and MC813-70 were screened against the ganglioside fractions from RBC, lymphocytes, neutrophils, monocytes, kidney, and brain. Consistent with the known distribution of MSGG, ${ }^{5,23,32}$ MC813-70+ and $\mathrm{B} 2+$ gangliosides were observed in RBCs and kidney. No bands were observed in any other tissues tested.

\section{MoAb B2 does not hemagglutinate human RBC}

The ability of MoAbs B2, RM1, and MC813-70 to agglutinate RBCs was tested against either washed or ficinmodified LKE-S RBC using standard conditions established for MoAb MC813-70. ${ }^{28,29}$ No MoAb tested was capable of agglutinating washed RBCs. As reported, ${ }^{29}$ MoAb MC813-70 strongly agglutinated ficin-modified LKE-S RBC. In contrast, neither MoAb B2 nor MoAb RM1 agglutinated ficin-treated RBCs, even after prolonged $4^{\circ} \mathrm{C}$ incubation (up to $4 \mathrm{hr}$ ), increasing the antibody-cell ratio and filter concentrating the antibody 50fold. Contrary to expectations, MoAb B2 is not a viable serologic reagent for LKE phenotyping.

\section{Differential reactivity of anti-MSGG-LKE MoAbs by flow cytometry}

The reactivities of MoAbs B2, RM1, and MC813-70 were also compared by flow cytometry (Fig. 3). All three MoAbs were tested against ficin-treated LKE-S RBCs and two, MSGG+ RCC lines, A498 and ACHN. MSGG-LKE is strongly expressed in some RCCs, where it is hypothesized to play a role in tumor growth and metastasis. ${ }^{35,36}$

As previously reported, ${ }^{28,29}$ strong MoAb MC813-70 binding was observed against LKE-S RBCs. Neither MoAb B2 nor MoAb RM1 stained RBCs. Differential staining was observed with the RCC lines. Although all three antibodies strongly bound ACHN cells, only MC813-70 significantly stained A498 cells. In A498 cells, B2 staining was decreased 50 percent and was essentially absent with MoAb RM1. These differences are reminiscent of other anti-carbohydrate MoAbs, which may recognize the same epitope but display different staining characteristics when tested against intact cell membranes. ${ }^{27,37}$

\section{DISCUSSION}

MSGG or LKE antigen is a high-incidence antigen (98\%$99 \%$ of donors) on human RBCs. ${ }^{28,29}$ Because it is a high-incidence RBC antigen, very few examples of human anti-LKE are described in the literature. ${ }^{38-40}$ Like anti- $\mathrm{P}^{\mathrm{k}}$ and $\mathrm{P}$ antibodies, examples of human anti-LKE are typically of IgM isotype, showing enhanced reactivity at $4^{\circ} \mathrm{C}$ and after protease digestion. ${ }^{38}$ Although human anti-LKE is not readily available, MoAb MC813-70, a murine MoAb 

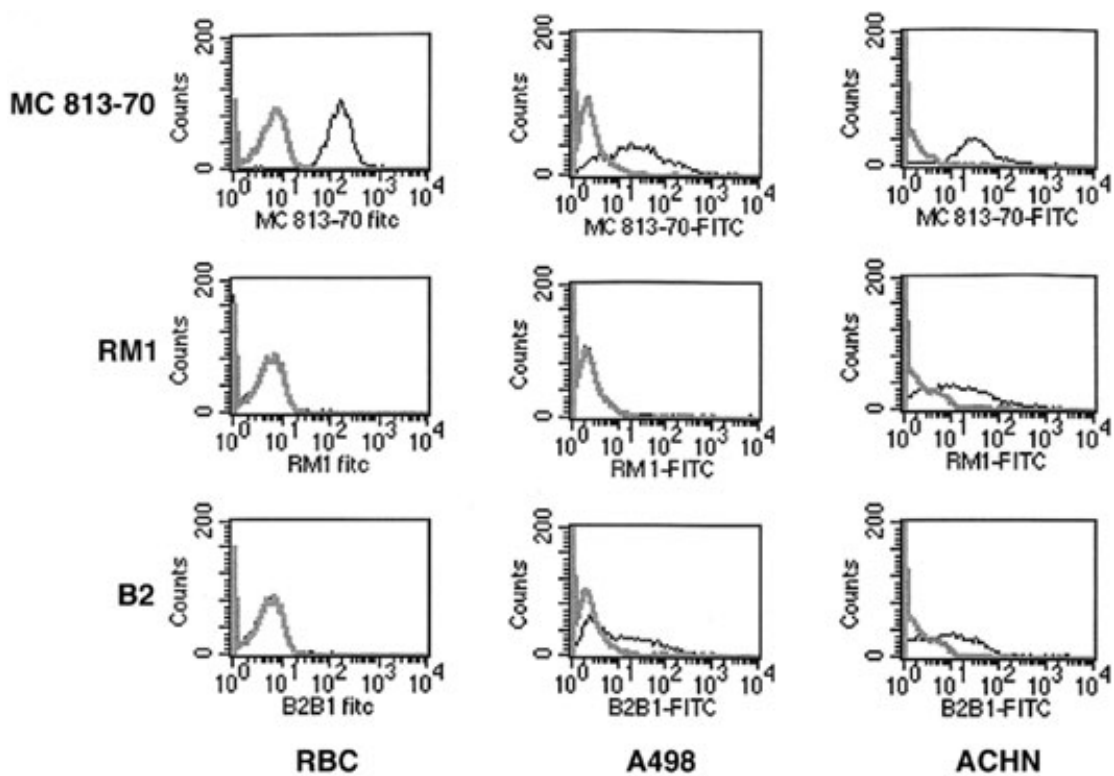

Fig. 3. MoAbs B2, MC813-70, and RM1 differ in their binding to cell membranes. MoAb MC813-70, RM1, and B2 were tested against LKE-S RBCs, A498, and ACHN RCCs cells by flow cytometry (dark line). Isotype control is also shown (gray line).
The identification of the $\mathrm{B} 2$ antigen as MSGG-LKE suggests a possible role for LKE in neural crest differentiation. It is well established that neurons of the sympathetic nervous system and adrenal chromaffin cells share a common embryonic heritage via the caudal neural crest. ${ }^{20-22} \mathrm{~A}$ transient marker on bipotential embryonic adrenal cells, LKE or B2 antigen signals early neuroblast commitment along sympathetic neural development. ${ }^{21}$ In the spinal cord, which also arises from the caudal neural crest, LKE, and its precursor $\mathrm{Gb}_{5}$ (Table 1), specifically identify subsets of myelinated afferent sensory neurons responsible for transmitting proprioception and touch. ${ }^{16-18}$ After synapsing in the spinal cord dorsal horn, these LKE+ neurons synapse with their corresponding sensory ganglia in the cerebellum. Like their dorsal horn counterparts, these afferent sensory ganglia cells are also LKE-positive. ${ }^{16,17}$

In summary, we have identified the B2 antigen as the globo-ganglioside, MSGG, or LKE antigen. Our findings suggest a potential role for MSGG in neuroendocrine differentiation, marking immature sympathetic neuroblasts and bipotential embryonic adrenal chromaffin cells. Unfortunately, MoAb B2 is not useful as a serologic reagent for RBC phenotyping. MoAb B2 may, however, be a useful reagent for embryonic stem cells, ${ }^{4,5}$ embryonic carcinoma cells, and RCCs, ${ }^{6,13,35,36}$ which strongly express MSGG or LKE antigen.

\section{ACKNOWLEDGMENTS}

We thank the laboratory of John Lowe, MD, for use of the flow cytometer, Yuan Gu, MD, for technical assistance, and Elizabeth Horn Walker for her help in figure design and composition.

\section{REFERENCES}

1. Hakomori S. Structure, organization, and function of glycosphingolipids in membrane. Curr Opin Hematol 2003;10:16-24.

2. Hakomori S. Cancer-associated glycosphingolipid antigens: their structure, organization and function. Acta Anat 1998;161:79-90.

3. Muller R, Altmann F, Zhou D, Hennet T. The Drosophila melanogaster brainiac protein is a glycolipid-specific $\beta 1,3$ $N$-acetylglucosaminyl-transferase. J Biol Chem 2002;277: 32417-20.

4. Thomson JA, Itskovitz-Eldor J, Shapiro SS, et al. Embryonic 
stem cell lines derived from human blastocysts. Science 1998;282:1145-7.

5. Shevinsky LH, Knowles BB, Damjanov I, Solter D. Monoclonal antibody to murine embryos defines a stagespecific embryonic antigen expressed on mouse embryos and human teratocarcinoma cells. Cell 1982;30:697-705.

6. Song Y, Withers DA, Hakomori S. Globoside-dependent adhesion of human embryonal carcinoma cells, based on carbohydrate-carbohydrate interaction, initiates signal transduction and induces enhanced activity of transcription factors AP1 and CREB. J Biol Chem 1998;273:2517-25.

7. Hennet T. The galactosyltransferase family. Cell Mol Life Sci 2002;59:1081-95.

8. Vollrath B, Fitzgerald KJ, Leder P. A murine homologue of the Drosophila brainiac gene shows homology to glycosyltransferases and is required for preimplantation development of the mouse. Mol Cell Biol 2001;21:5688-97.

9. Fenderson BA, Andrews PW, Nudelman E, Clausen H, Hakomori S. Glycolipid core structure switching from globoto lacto- and ganglio-series during retinoic acid-induced differentiation of TERA-2-derived human embryonal carcinoma cells. Dev Biol 1987;122:21-4.

10. Chen C, Fenderson BA, Andrews PW, Hakomori S. Glycolipid glycosyltransferases in human embryonal carcinoma cells during retinoic acid induced differentiation. Biochemistry 1989;28:2229-38.

11. Cooling LLW, Zhang DS, Naides SJ, Koerner TAW. Glycosphingolipid expression in acute nonlymphocytic leukemia: common expression of shiga toxin and parvovirus B19 receptors on early myeloblasts. Blood 2003;101: 711-21.

12. Cooling LLW, Koerner TAW, Naides SJ. Multiple glycosphingolipids determine the tissue tropism of parvovirus B19. J Infect Dis 1995;172:1198-205.

13. Krupnick JG, Damjanov I, Damjanov A, Zhu ZM, Fenderson BA. Globo-series carbohydrate antigens are expressed in different forms on human and murine tertocarcinomaderived cells. Int J Cancer 1994;59:692-8.

14. Ariga T, Yu RK. The role of globo-series glycolipids in neuronal cell differentiation-a review. Neurobiochem Res 1998;23:291-303.

15. Zurn AD. Identification of glycolipid binding sites for soybean agglutinin and differences in the surface glycolipids of cultured adrenergic and cholinergic sympathetic neurons. Dev Biol 1982;94:483-98.

16. Dodd J, Solter D, Jessell TM. Monoclonal antibodies against carbohydrate differentiation antigens identify subsets of primary sensory neurones. Nature 1984;311:469-72.

17. Dodd J, Jessell TM. Lactoseries carbohydrates specify subsets of dorsal root ganglion neurons projecting to the superficial dorsal horn of rat spinal cord. J Neurosci 1985;5:3278-94.

18. Holford C, Case P, Lawson SN. Substance P, neurofilament, peripherin and SSEA4 immunocytochemistry of human dorsal root ganglion neurons obtained from post-mortem tissue: a quantitative morphometric analysis. Neurocytology 1994;23:577-89.

19. Ariga T, Ando S, Takashashi A, Miyatake T. Gangliosides and neutral glycolipids of human adrenal medulla. Biochim Biophys Acta 1980;618:480-5.

20. Carnham JR, Patterson PH. The generation of monoclonal antibodies that bind preferentially to adrenal chromaffin cells and the cells of embryonic sympathetic ganglia. J Neurosci 1991;11:3493-506.

21. Anderson DJ, Carnahan JF, Michelsohn A, Patterson PH. Antibody markers identify a common progenitor to sympathetic neurons and chromaffin cells in vivo and reveal the timing of commitment to neuronal differentiation in the sympathoadrenal lineage. J Neuroscience 1991;11:3507-19.

22. Anderson DJ, Axel R. A bipotential neuroendocrine precursor whose choice of cell fate is determined by NGF and glucocorticoids. Cell 1986;7:1079-90.

23. Kannagi R, Cochran NA, Ishigami F, et al. Stage-specific embryonic antigens (SSEA-3 and -4) are epitopes of a unique globo-series ganglioside isolated from human teratocarcinoma cells. EMBO J 1983;2:2355-61.

24. Kannagi R, Levery SB, Ishigami F, et al. New globoseries glycosphingolipids in human teratocarcinoma reactive with the monoclonal antibody directed to a developmentally regulated antigen, stage-specific embryonic antigen 3. J Biol Chem 1983;258:8934-42.

25. Kannagi R, Nudelman E, Levery SB, Hakomori S. A series of human erythrocyte glycosphingolipids reacting to the monoclonal antibody directed to a developmentally regulated antigen, SSEA-1. J Biol Chem 1982;257:14865-74.

26. Fenderson BA, Nichols EJ, Clausen H, Hakomori SI. A monoclonal antibody defining a binary $\mathrm{N}$-acetyllactosaminyl structure in lactoisooctaosyl ceramide. Mol Immunol 1986;23:747-54.

27. Saito S, Levery SB, Salyan MEK, Goldberg RI, Hakomori S. Common tetrasaccharide epitope NeuAc $\alpha 2-3 \mathrm{Gal} \beta 1-3$ (NeuAco2-6) GalNAc, presented by different carrier glycosylceramides or O-linked peptides, is recognized by different antibodies and ligands having distinct specificities. J Biol Chem 1994;269:5644-52.

28. Cooling LL, Kelly K. Inverse expression of $\mathrm{P}^{\mathrm{k}}$ and Luke blood group antigens on human RBCs. Transfusion 2001;41:898907.

29. Tippet P, Andrews PW, Knowles BB, Solter D, Goodfellow PN. Red cell antigens P (globoside) and Luke: identification by monoclonal antibodies defining the murine stagespecific embryonic antigens-3 and -4 (SSEA-3 and SSEA-4). Vox Sang 1986;51:53-6.

30. Judd JW. Methods in immunohematology. 2nd ed. Durham, (NC): Montgomery Scientific; 1994.

31. Ledeen RW, Yu RK. Gangliosides: structure, isolation and analysis. Methods Enzymol 1982;83:139-91.

32. Cooling LW, Zhang DS, Koerner TA. Human platelets express gangliosides with LKE activity and ABH blood group activity. Transfusion 2001;41:504-16. 
33. Prokazova NV, Orekhov AN, Mukhin DN, et al. The gangliosides of adult human aorta: intima, media and plaque. Eur J Biochem 1987;167:349-52.

34. Koerner TA, Weinfeld HM, Bullard LS, Williams LC. Antibodies against platelet glycosphingolipids: detection in serum by quantitative HPTLC-autoradiography and association with autoimmune and alloimmune processes. Blood 1989;74:274-84.

35. Katagiri YU, Ohmi K, Katagiri C, et al. Prominent immunogenicity of monosialosyl galactosylgloboside, carrying a stage-specific embryonic antigen-4 (SSEA-4) epitope in the ACHN human renal tubular cell line-a simple method for producing monoclonal antibodies against detergent-insoluble microdomains/raft. Glycoconjugate J 2001;18:347-53.

36. Saito S, Nojiri H, Satoh M, Ito A, Ohyama C, Orikasa S.
Inverse relationship of expression between GM3 and globoseries ganglioside in human renal cell carcinoma. Tohoku J Exp Med 2000;190:271-8.

37. Dohi T, Nemoto T, Ohta S, et al. Different binding properties of three monoclonal antibodies to sialyl LeX glycolipids in a gastric cancer cell line and normal stomach tissue. Anticancer Res 1993;13:1277-782.

38. Tippet P, Sanger R, Race RR, Swanson J, Busch S. An agglutinin associated with the $\mathrm{P}$ and the $\mathrm{ABO}$ blood group systems. Vox Sang 1965;10:269-80.

39. Moller B, Jorgensen J. Phenotype frequency of LKE in the Danish population. Hum Hered 1988;38:375-7.

40. Bruce M, Watt A, Gabra GS, Ritchell R, Lakhesar D, Tippet P. LKE red cell antigen and its relationship to $\mathrm{P}_{1}$ and $\mathrm{P}^{\mathrm{k}}$ : serological study of a large family. Vox Sang 1988;55:23740. 凹 